\title{
Hypothermia Attenuates $\beta 1$ Integrin Expression on Extravasated Neutrophils in an Animal Model of Meningitis
}

\author{
Mark E. Rowin, ${ }^{1}$ Vivian Xue, ${ }^{1}$ and Jose Irazuzta $^{1}$
}

\begin{abstract}
Brain injury in meningitis occurs in part as a consequence of leukocyte migration and activation. Leukocyte integrins are pivotal in the inflammatory response by mediating adhesion to vascular endothelium and extracellular matrix proteins. We have demonstrated that moderate hypothermia early in the course of meningitis decreases leukocyte sequestration within the brain parenchyma. This study examines whether hypothermia alters neutrophil integrin expression in a rabbit model of bacterial meningitis. Prior to the induction of meningitis, peripheral blood samples were obtained and the neutrophils isolated. Sixteen hours after inducing group B streptococcal meningitis, animals were treated with antibiotics, IV fluids, and mechanically ventilated. Animals were randomized to hypothermia $\left(32-33^{\circ} \mathrm{C}\right)$ or normothermia conditions. After 10 hours of hypothermia or normothermia, neutrophils were isolated from the blood and cerebral spinal fluid (CSF), stained for $\beta 1$ and $\beta 2$ integrins, and analyzed using flow cytometry. Cerebral spinal fluid neutrophil $\beta 1$ integrin expression was significantly decreased in hypothermic animals. Beta-1 integrins can assume a higher affinity or "activated" state following inflammatory stimulation. Expression of "activated" $\beta 1$ integrins was also significantly decreased in hypothermic animals. Beta2 CSF neutrophil integrin expression was decreased in hypothermic animals, but failed to reach significance. These data suggest hypothermia may attenuate extravasated leukocyte expression of both total and "activated" $\beta 1$ integrins.
\end{abstract}

KEY WORDS: integrin; hypothermia; meningitis; neutrophils.

\section{INTRODUCTION}

The process of inflammation is a complex interplay of agonists, cytokines, endothelial cells, and myeloid cells. Polymorphonuclear leukocytes (PMNs) are important in cellular defense of the host by releasing oxygen radicals and lysosomal enzymes necessary for the eradication of invading microorganisms. Although PMNs play a vital role in host defense, the accumulation of activated PMNs at sites of tissue injury coupled with the excess release of bio-active products may increase tissue injury (1-8). Recently, specific therapies designed to attenuate excessive leukocyte activation have been examined. In inflammatory brain injury, blockade of neutrophil sequestration

${ }^{1}$ Division of Pediatric Critical Care Medicine, Children's Hospital Medical Center, 3333 Burnet Avenue, Cincinnati, Ohio 45229-3039. and activation decreases PMN-mediated tissue destruction (9-11).

Integrins are cell adhesion molecules that play a primary role in leukocyte sequestration. Integrins are heterodimeric glycoproteins consisting of two non-covalently linked chains, the $\alpha$ and $\beta$ subunits. Integrins are commonly classified according to their $\beta$ subunit. The major class of integrins present on PMNs are $\beta 2$ integrins $(\alpha \mathrm{L} \beta 2$ or LFA-1; $\alpha \mathrm{M} \beta 2$ or Mac-1; and $\alpha \mathrm{X} \beta 2$ or p150,95) (12). Upon activation, $\beta 2$ integrins mediate firm PMN adhesion to endothelium and subsequent transmigration of the leukocyte out of the vasculature. $\beta 1$ integrins have also been described on PMNs. They are a minority of the integrin population and their role is poorly understood. Many researchers have speculated that the role of $\beta 1$ integrins on human 
PMN is as receptors for extracellular matrix macromolecules. Polymorphonuclear leukocytes express $\beta 1$ integrins that recognize a domain on the extracellular matrix proteins fibronectin $(\alpha 5 \beta 1)$ and laminin $(\alpha 6 \beta 1)$ (13-14). Recently, researchers have demonstrated that the $\beta 1$ integrin plays a role in binding of PMNs to TNFstimulated endothelial cells $(\alpha 4 \beta 1)$ (15), or expressed following transmigration through endothelial cells or a fibroblast layer $(\alpha 4, \alpha 5$ and $\alpha 6 \beta 1)$ (16-18).

Integrins may also play a role in infectious or inflammatory disease processes. Certain bacterial and viral infections are known to alter expression of $\beta 1$ and $\beta 2$ integrin subpopulations on both circulating granulocytes and lymphocytes (19-21). During inflammation, $\beta 1$ integrins appear to play a critical role in directed lymphocyte migration. The $\alpha 4 \beta 1$ integrin on T cells assists in lymphocyte recruitment into the subarachnoid space during viral meningitis (22). HIV infection causes increased expression of $\alpha 5 \beta 1$ integrins on both acutely and chronically infected CD4+ T cells, with resultant increased adherence to extracellular matrix glycoproteins (23-24).

Multiple investigators have shown that hypothermia decreases PMN accumulation in animal models of brain injury (25-28). We have recently shown that hypothermia may be of benefit in the treatment of meningitis. The application of moderate hypothermia decreases brain myeloperoxidase levels, CSF nitric oxide, and central nervous system (CNS) excitatory amino acid levels (29-30). We have previously demonstrated that extravasated CSF neutrophils in rabbits with group B streptococcal meningitis show a significant increase in expression of $\beta 1$, activated $\beta 1$, and $\beta 2$ integrins (31). The mechanism(s) whereby hypothermia diminishes CNS injury remains unknown. Since the brain injury in meningitis occurs in part as a consequence of leukocyte activation and subsequent release of bioactive products, we speculate that hypothermia affects neutrophil function during the acute inflammatory response. To our knowledge, the effects of hypothermia on neutrophil integrin expression during meningitis have not been explored. Thus, this article examines expression of $\beta 1$ and $\beta 2$ integrins on extravasated neutrophils in normothermic and hypothermic animals using bacterial meningitis as a model of CNS inflammation.

\section{MATERIALS AND METHODS}

Materials. Monoclonal antibody (MAb) 15/7 was provided by Dr. Ted Yednock (Athena NeuroSciences,
San Francisco, California). Monoclonal antibody 15/7 is a murine anti-human $\operatorname{IgG}$ that binds to an activationdependent epitope expressed on the $\beta$ subunit of $\beta 1$ integrins (32). The anti- $\beta 2$ IgG monoclonal antibody $(\mathrm{mAb} 1388)$ and the anti- $\beta 1 \mathrm{IgG}$ monoclonal antibody (MAb 1981) were purchased through Chemicon Inc. (Temecula, California). Both of these antibodies react with the extracellular domains of their respective integrins. The hybridoma secreting the murine MAb dA4.4 $\left(\mathrm{IgG}_{1}\right)$ directed against the $\mu$ chain of human $\operatorname{IgM}$ was obtained through American Type Tissue Culture Collection (Rockville, Maryland). Monoclonalantibody dA4.4 was purified from ascites by octanoid acid precipitation and was provided by Dr. John Bohnsack (University of Utah).

The following reagents were purchased: Hank's buffered saline (HBSS) (Gibco BRL, Gaithersburg, Maryland), purified rat immunoglobulin G (Calbiochem-Novabiochem Corporation, La Jolla, California), phycoerythrin-conjugated $\mathrm{F}\left(\mathrm{ab}^{\prime}\right)_{2}$ goat anti-mouse $\mathrm{IgG}$ and phycoerythrin-conjugated $\mathrm{F}\left(\mathrm{ab}^{\prime}\right)_{2}$ goat anti-rat IgG (Immunotech, Miami, Florida).

Animal Model. Male New Zealand rabbits (3-4 $\mathrm{kg}$ ) were used. Standard animal husbandry and care practices were employed. A preliminary blood sample was obtained prior to induction of meningitis.

To induce meningitis, a 23-gauge needle was introduced in the cisterna magnae and $0.3 \mathrm{ml}$ of cerebral spinal fluid was withdrawn. After removal of CSF, the animal's cisterna magnae was inoculated with $0.3 \mathrm{ml}$ of a suspension containing $1 \times 10^{5} \mathrm{cfu} / \mathrm{ml}$ of Group B Streptococcus (GBS), serotype III, diluted in sterile normal saline. Sixteen hours post-inoculation the rabbits were treated with i.v. Ceftriaxone $(50 \mathrm{mg} / \mathrm{kg})$. The rabbits were then anesthetized and a tracheostomy was performed for continuous mechanical ventilation. The animals then underwent tracheotomy and continuous mechanically ventilated. Animals were randomized to hypothermic (defined as 32-33C) vs. normothermic (defined as 37-39C) conditions. Animals in the hypothermia group were rapidly cooled by covering their torso with a plastic bag containing ice. Temperatures were maintained within set parameters with the use of warming/cooling blankets. Intravenous fluids were administered over the next $10 \mathrm{~h}$. Blood pressure, heart rate, and temperature were continuously monitored. $\mathrm{PaO} 2$ and $\mathrm{PaCO} 2$ were monitored every $2 \mathrm{~h}$.

A second cisternal tap was performed after $10 \mathrm{~h}$ of hypothermia/normothermia and $1 \mathrm{ml}$ of CSF was withdrawn. A concurrent blood sample was obtained at 
the time of the second cisterna magnae puncture. Neutrophils were isolated as previously described (14). CSF samples were centrifuged and the cell pellet was resuspended in buffered saline and evaluated for integrin expression.

Flow Cytometry. Neutrophils from peripheral blood and CSF samples were isolated within $30 \mathrm{~min}$ of collection and viability confirmed with trypan blue exclusion. Neutrophils were harvested using dextran sedimentation and centrifugation through Ficoll Hypaque (14). Peripheral and CSF PMN samples were washed, centrifuged, and re-suspended in buffered saline solution to a concentration of $1 \times 10^{6}$ cells $/ \mathrm{ml}$. Viability of all cells was confirmed by trypan blue exclusion and was consistently greater than $95 \%$. Histologic evaluation using direct hemocytometric observation was performed on all samples to confirm cells were predominantly PMNs. Cells were incubated with saturating concentrations of monoclonal antibodies directed towards the CD 18 ( $\beta 2)$ subunit, CD 29 ( $\beta 1)$ subunit, or an activation-dependent epitope located on the ligand recognition domain of the $\beta 1$ subunit. PMNs isolated from the CSF were also stained with isotype matched controls to detect nonspecific binding. Cells were then washed, counterstained with a phycoerythrin-conjugated IgG antibody and finally fixed with $1 \%$ paraformaldehyde in PBS, pH 7.4. Labeled cells were analyzed on a FACScaliber flow cytometer (Becton Dickinson Inc., San Jose, California). Neutrophil populations were identified and gated by forward vs. right angle light scatter characteristics. Cells, $\left(10^{4}\right)$ per gated determination were measured and mean channel fluorescence was analyzed. Using available software, the mean fluorescence of the cell distribution was calculated (FACScan ${ }^{\circledR}$, Becton Dickinson, Mountain View, California). Relative fluorescence intensity was defined as the ratio of mean fluorescence of labeled CSF PMNs (after correction for nonspecific binding) compared to the fluorescence of circulating PMNs.

Total neutrophil counts were performed on blood and CSF samples by FACScan ${ }^{\circledR}$ analysis. Neutrophils were identified by size characteristics and nucleus to cytoplasm ratios, and expressed as number of cells per deciliter.

Statistical Evaluation. All values are expressed as mean \pm SEM of $N$ observations, where $N$ represents the number of animals. Significance is declared for $P<0.05$. For comparison of two groups, Student's paired $t$-tests are used. When multiple variables were studied, all data was corrected for multiple comparisons. All statistics were performed using a commercially available statistical program (StatView 5.0; Abacus Concepts, Inc.) on a Power G 3 Macintosh.

\section{RESULTS}

The integrin family is strongly conserved across vertebrate species (33-35). Multiple investigators have used anti-human integrin antibodies in rabbit models of various in vivo and in vitro experiments [36-40]. We have previously shown the antibodies used in this experiment recognize rabbit integrin structures $(31,41-42)$.

Data were collected from 16 animals with group B streptococcal meningitis ( 8 in hypothermia group, 8 in normothermia group). As part of our experiment, we initially evaluated whether hypothermia affects the amount of neutrophil extravasation into the subarachnoid space. Animals exposed to hypothermia for $10 \mathrm{~h}$ had a CSF WBC count of 1662 cell/dl while normothermic animals had a CSF WBC count of 897 cell/dl. The increase in CSF neutrophils did not reach significance between these two groups $(P=0.1)$. Similarly, there was no difference in the absolute neutrophil count in the peripheral circulation between normothermic and hypothermic animals (hypothermia $=11.4 \times 10^{3}$ cells $/ \mathrm{dl}$ vs., normothermia $=$ $10.0 \times 10^{3}$ cells $\left./ \mathrm{dl}\right)$.

Flow cytometry data is expressed as relative fluorescence intensity (RFI) and is defined as the ratio of mean channel fluorescence of antibody stained-cells compared to fluorescence at baseline. Baseline is defined as expression of the specific integrin on circulating neutrophils, prior to induction of meningitis. All samples were corrected for fluorescence secondary to nonspecific binding.

We examined whether hypothermia affects total expression of $\beta 1$ integrins on CSF neutrophils. Expression of total cell surface $\beta 1$ integrins in normothermic animals increased from a baseline measurement of 1.03 to $1.92(P<0.01)$. As is shown in Figure 1 , hypothermia significantly decreased total $\beta 1$ integrin expression on neutrophils when compared to normothermic animals, with the relative fluorescence intensity decreasing to near baseline levels (RFI $=1.18, P<0.05$ ).

In addition to total surface number, $\beta 1$ integrins are able to change their quartinary structure and assume a higher affinity or activated state. This change in affinity modulation can be detected by antibody recognition of a neoepitope expressed on the $\beta 1$ integrin ligand recognition domain. As shown in Figure 2, hypothermia sig- 


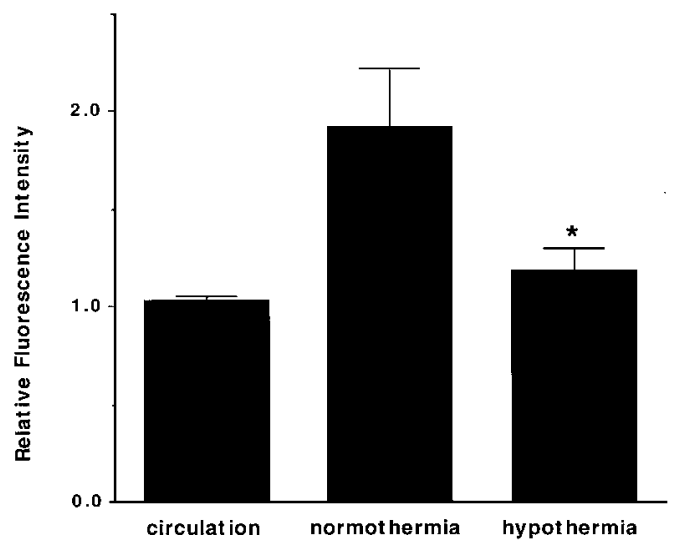

Fig. 1. Hypothermia significantly decreased $\beta 1$ integrin expression on extravasated cerebral spinal fluid neutrophils in animals with meningitis. Flow cytometric analysis demonstrates that the neutrophils in the peripheral circulation have a $\beta 1$ integrin expression of $1.03 \pm .02$. $\beta 1$ integrin expression on spinal fluid neutrophils in normothermic animals increased to $1.92 \pm 0.3$. In animals exposed to $10 \mathrm{~h}$ of hypothermia, $\beta 1$ integrin expression significantly decreased to $1.18 \pm .12\left({ }^{*} \mathrm{P}<0.05\right)$. Relative fluorescence intensity is the ratio of mean channel fluorescence of labeled neutrophils compared to PMNs in circulation prior to induction of meningitis. Mean channel fluorescence of extravasated PMNs is corrected for nonspecific binding prior to analysis. The data represent the means $\pm S E M$ of 8 animals in each condition.

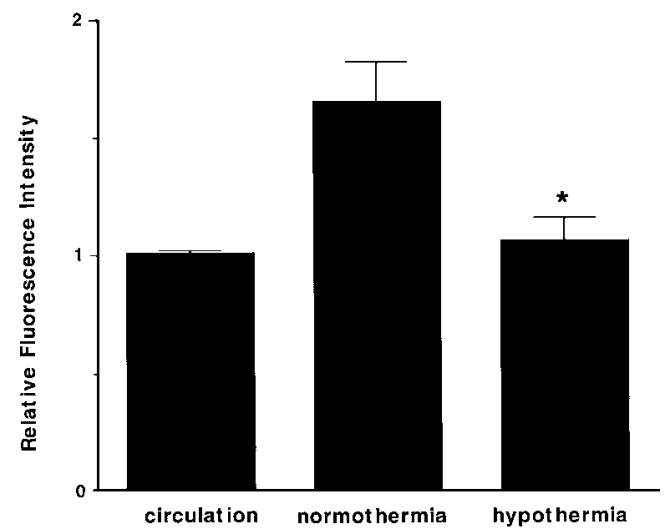

Fig. 2. Hypothermia significantly decreased expression of a $\beta 1$ integrin activation-dependent epitope on extravasated cerebral spinal fluid neutrophils in animals with meningitis. Flow cytometric analysis of the epitope recognized by MAb 15/7 demonstrated a baseline binding of $1.01 \pm .01$. Binding of MAb $15 / 7$ on spinal fluid neutrophils in normothermic animals increased to $1.65 \pm 0.17$. In animals exposed to $10 \mathrm{~h}$ of hypothermia, binding of MAb 15/7 significantly decreased to $1.06 \pm .1\left({ }^{*} P=0.01\right)$. Relative fluorescence intensity is the ratio of mean channel fluorescence of labeled neutrophils compared to PMNs in circulation prior to induction of meningitis. Mean channel fluorescence of extravasated PMNs is corrected for nonspecific binding prior to analysis. The data represent the means $\pm S E M$ of 8 animals in each condition.

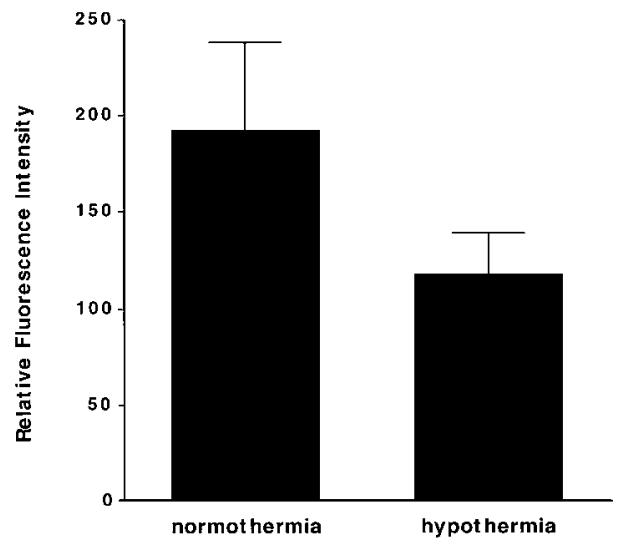

Fig. 3. $\beta 2$ integrin expression on extravasated cerebral spinal fluid neutrophils in hypothermic animals with meningitis decreased but failed to reach significance. Flow cytometric analysis demonstrated the neutrophils in the peripheral circulation have a $\beta 2$ integrin expression of $1.06 \pm .05$. Concurrent $\beta 2$ integrin expression on neutrophils in spinal fluid increased to $192 \pm 46$ in normothermic animals. In animals exposed to 10 hours of hypothermia, $\beta 2$ integrin expression decreased to $117 \pm 23$, but failed to reach significance $(P=0.11)$. Relative fluorescence intensity is the ratio of mean channel fluorescence of labeled neutrophils compared to PMNs in circulation prior to induction of meningitis. Mean channel fluorescence of extravasated PMNs is corrected for nonspecific binding prior to analysis. The data represent the means $\pm S E M$ of 8 animals in each condition.

nificantly decreased expression of the activation-dependent epitope located on the $\beta 1$ integrin and recognized by MAb 15/7. Relative fluorescence intensity decreased from 1.65 to $1.06(P=0.01)$. These data suggest that hypothermia decreases total cell surface expression, as well as affinity modulation of the $\beta 1$ integrin receptor.

The largest percent of the neutrophil integrin population are the CD-18 ( $\beta 2$ integrin) receptors. Figure 3 compares expression of $\beta 2$ integrins on extravasated CSF neutrophils. A 39\% decrease in relative fluorescence intensity was noted in extravasated PMNs exposed to hypothermic conditions. However, this decrease failed to reach significance $(P=0.11)$.

We also evaluated whether hypothermic treatment of meningitis changes integrin expression on circulating neutrophils. $\beta 1$, activated $\beta 1$, and $\beta 2$ integrin expression prior to and following 10 hours of hypothermia did not significantly change on the circulating neutrophil population (Table 1). Regression analysis was performed on all three integrin groups studied and showed no relationship between changes in integrin expression on peripheral neutrophils and concurrently obtained extravasated neutrophils. 
Table 1. Integrin Expression.

\begin{tabular}{lccc}
\hline & Normothermia & Hypothermia & $\mathrm{p}$ value \\
\hline$\beta 1$ Integrin & $1.03 \pm .02$ & $1.05 \pm .03$ & .55 \\
"Activated" $\beta 1$ Integrin & $1.01 \pm .01$ & $1.00 \pm .01$ & .52 \\
$\beta 2$ Integrin & $1.08 \pm .05$ & $1.01 \pm .02$ & .10 \\
\hline
\end{tabular}

Integrin expression on circulating neutrophils did not significantly change as a consequence of hypothermia during meningitis. Integrin expression on peripheral neutrophils was evaluated by flow cytometric analysis. Numbers are expressed as relative fluorescence intensity (RFI) units. Relative fluorescence intensity is the ratio of mean channel fluorescence of circulating neutrophils after $10 \mathrm{~h}$ of hypothermia or normothermia during meningitis compared to baseline neutrophil fluorescence. Baseline expression is defined as integrin expression prior to the induction of meningitis. The data represent the means $\pm \mathrm{SD}$ of 7 animals.

\section{DISCUSSION}

Hypothermia has been documented in numerous animal models to decrease PMN accumulation within injured or inflamed brain tissue (25-28). Hypothermia decreased intracranial swelling and improved morbidity in severe head trauma (43-45). Hypothermia also decreased PMN accumulation following ischemia and reperfusion injury (46). We have previously shown that hypothermia decreased PMN accumulation within the brain parenchyma during acute bacterial meningitis. In this rabbit model of bacterial meningitis, myeloperoxidase concentration within brain tissue decreased 35\% in the hypothermic group (29). The temperature-dependent cellular signal responsible for decreasing PMN accumulation has not been defined. Our data suggest that surface integrin expression may be one of the cellular pathways affected by hypothermia. Both total and activated $\beta 1$ integrin expression on exravasated neutrophils decreased to near baseline levels following 10 hours of hypothermia during meningitis. Integrin $\beta 2$ expression on extravasated PMNs also decreased, but failed to reach significance. In our model, hypothermia does not affect integrin expression on the circulating neutrophil population.

The physiologic mechanism(s) that are responsible for the neuro-protective effect of hypothermia are poorly understood. Originally, CNS hypothermia was believed to preserve the high energy phosphate stores and to decrease cerebral metabolism (47). However, the beneficial effects from hypothermia appear to be secondary to suppression of the inflammatory cascade and not to a delay in the injury process (48). In our model of group B streptococcal meningitis, hypother- mia decreased the CSF concentrations of glutamate and aspartate by $40-50 \%$ and caused a significant decrease in the induction of $72-\mathrm{kDa}$ heat shock protein (30). In a traumatic brain injury model in rats. Whalen et al. demonstrates hypothermia decreased neutrophil accumulation within the injured brain tissue (25). This effect was independent of endothelial E-selectin or ICAM-1 expression (26). Our findings suggest one mechanism that may account for the decrease in neutrophil accumulation is the down-regulation of both total and activated $\beta 1$ integrin expression on extravasated neutrophils.

The beneficial effects induced by hypothermia were not secondary to modification of the degree of infection or bacterial load. There was no difference in the number of positive CSF cultures between groups at the end of the observation period. One CSF sample from each group grew GBS.

The effects of hypothermia on circulating PMNs are well described. In a rabbit model of hypothermia, circulating neutrophil counts significantly increased, but did not exhibit increased oxygen burst or phagocytic activity (49). In human studies, many of the studies evaluating the effects of hypothermia on granulocyte physiology and adhesion are reported in the cardiopulmonary bypass literature. Hypothermia during cardiopulmonary bypass is associated with an initial decrease in circulating PMNs, followed by a marked increase that persisted up to 24 hours into the postoperative period (50). This effect appears to be independent of hypothermia-induced changes in endothelial-derived adhesion molecules (51). Neutrophil activation, as measured by serum elastase release, decreases during hypothermic cardiopulmonary bypass (52). There are few studies in the literature with regards to the effects of hypothermia on integrin expression. Finn et al. reported that in children undergoing cardiopulmonary bypass, a gradual rise in $\beta 2$ expression on neutrophils was noted in a small segment of the granulocyte population (53). Our studies showed no significant change in $\beta 2$ integrin expression on circulating neutrophils as a consequence of hypothermia in meningitis. Paugam et al. reported that $\beta 2$ expression on neutrophils increased within 20 min of cardiopulmonary bypass. However, this rapid increase in $\beta 2$ expression was independent of both temperature and interleukin-8 production (54).

On neutrophils, $\beta 2$ integrins facilitate firm binding to activated endothelium. While $\beta 1$ integrins on neutrophils are known to function as an extracellular protein adhesion molecule, their role may be more complex. Several clinical and laboratory observations suggest $\beta 2$ 
integrin-independent mechanisms may contribute to neutrophil recruitment and activation in infectious states. PMNs have been found post-mortem in the alveolar spaces of a patient with $\beta 2$ integrin deficiency (leukocyte adhesion deficiency I) (55). Hepatic sequestration of neutrophils in a rat model of endotoxemia has been shown to be $\beta 2$ integrin-independent (56). Migration of PMN into the alveolar space in both pneumonia and chemical lung injury models is not inhibited by anti- $\beta 2$ monoclonal antibody $(57,58)$. Similarly, anti- $\beta 2$ integrin monoclonal antibody did not effect mortality nor total CSF white blood cell count in a rat model of Haemophilus meningitis (59). We have previously shown a marked increase in $\beta 1$ (total and activated) and $\beta 2$ integrin expression on extravasated neutrophils when compared to concurrent expression on circulating neutrophils in GBS meningitis. Additionally, $\beta 1$ integrin expression on extravasated neutrophils demonstrated a significant correlation with CNS tissue PMN sequestration and blood brain barrier dysfunction (31). These studies suggest $\beta 1$ integrins play an important role in the pathophysiologic consequences of PMN-induced tissue injury.

This study was designed to evaluate the effect of short-term hypothermia on in vivo expression of $\beta 1$ and $\beta 2$ integrins on extravasated CSF neutrophils. We have previously shown that $\beta 1$, activated $\beta 1$ and $\beta 2$ integrins on extravasated neutrophils significantly increase during group B streptococcal meningitis (31). We now demonstrate that hypothermia significantly decreases $\beta 1$ integrin expression and activation on extravasated neutrophils. This effect is independent of total CSF WBC count or alteration in integrin expression on the circulating neutrophil population. In our model of GBS meningitis, we note the absolute value of extravasated neutrophils in hypothermic animals was higher than in normothermic animals, but did not reach statistical significance. We speculate this finding may be secondary to the model design. Meningitis was induced 16 hours prior to condition randomization. Animals randomized to hypothemic conditions demonstrated down-regulation of $\beta 1$ integrin number and function. Integrin $\beta 1$ on neutrophils are known to bind to extracellular proteins, thereby allowing directed migration. Thus, extravasated neutrophils in these animals may have impaired chemotaxis and sequester within CSF.

Alteration in integrin expression on extravasted neutrophils during hypothermia is a descriptive finding. The exact cellular signaling mechanism(s) affected by hypothermia is uncertain. Multiple pro-inflammatory chemokines are produced during bacterial meningitis. CSF concentrations of $\mathrm{TNF} \alpha$, IL- $8, \mathrm{GRO} \alpha$, MCP-1, MIP- $1 \alpha$ and MIP- $1 \beta$ all increase early in the time course of meningitis (60-61). These cytokines activate leukocytes and cause an increase in adhesion molecule expression. Additional studies to examine whether hypothermia affects the levels of these cytokines and whether they correlate with changes in expression of $\beta 1$ integrins are ongoing.

The relevance of integrin expression on neutrophils in specific infections is still under investigation. As PMNs perform the majority of their phagocytic functions outside the circulation, they must adhere to extracellular matrix (ECM) macromolecules in a regulated, reversible fashion in order to crawl through the tissue to the site of inflammation or infection. Better understanding the function of neutrophil integrin expression at sites of acute inflammation and the effects of hypothermia may suggest novel approaches to control the cascade of PMN-mediated pathophysiologic consequences associated with such processes as septic shock, ARDS, or the poor sequelae from meningitis.

Acknowledgments - This work was supported in part by a grant in aid from The Children's Medical Center Research Foundation (\#A014).

\section{REFERENCES}

1. Hansbrough, J. F., T. Wikstrom, M. Braide, M. Tenehaus, O. H. Rennekampee, V. Kiessig, and L. M. Bjursten. 1996. Neutrophil activation and tissue neutrophil sequestration in a rat model of thermal injury. J. Surg. Res. 61:17-22.

2. Matthay, M. A., H. G. Folkesson, A. Campagna, and F. Kheradmand. 1993. Alveolar epithelial barrier and acute lung injury. New Horizons 1:613-622.

3. Miota, J. M., T. J. Williams, P. G. Hellewell, and P. K. Jeffery. 1996. A role for the beta 2 integrin CD11b in mediating experimental lung injury in mice. Am. J. Respir. Cell Mol. Biol. 14:363-373.

4. Jaeschke, H., A. Farhood, and C. W. Smith. 1990. Neutrophils contribute to ischemia-reperfusion injury in rat liver in vivo. FASEB J. 4:3355-3359.

5. Granert, C., J. Raud, X. Xie, L. Lindquist, and L. Lindbom. 1994. Inhibition of leukocyte rolling with polysaccharide fucoidin prevents pleocytosis in experimental meningitis in the rabbit. J. Clin. Invest. 93:929-936.

6. Kornelisse, R. F., R. De Groot, and H. J. Neijens. 1995. Bacterial meningitis: mechanisms of disease and therapy. Eur. J. Pediatr. 154:85-96.

7. Hansen, P. R., and G. Stawski. 1994. Neutrophil mediated damage to isolated myocytes after anoxia and reoxygenation. Cardiovasc. Res. 28:565-569.

8. Leib, S., Y. Kim, L. Chow, R. Sheldon, and M. Tauber. 1996. Reactive oxygen intermediates contribute to necrotic and apototic 
neuronal injury in an infant rat model of bacterial meningitis due to Group B Streptococcus. J. Clin. Invest. 98:2632-2639.

9. Tuomanen, E., and A. Baruch. 1989. New antibodies as adjunctive therapies for gram positive bacterial meningitis. Pediatr. Infect. Dis. J. 8:923-928.

10. Vedder, N., R. Winn, C. Rice, Y. Chi, K. Arfors, and J. Harlan. 1988. A monoclonal antibody to the adherence-promoting leukocyte glycoprotein CD-13 reduces organ injury and improves survival from hemorrhagic shock and resuscitation in rabbits. J. Clin. Invest. 81:939-944.

11. Saez-Llorens, X., H. S. Jafari, F. Severien, K. D. Olsen, E. J. Hansen, I. I. Singer, and G. H. McCracken. 1991. Enhanced attenuation of meningeal inflammation and brain edema by concomitant administration of anti-CD18 monoclonal antibodies and dexamethasone in experimental Haemophilus meningitis. J. Clin. Invest. 88:2003-2011.

12. Arnaout, M. A. 1990. Structure and function of the leukocyte adhesion molecules CD11/CD18. Blood. 75:1037-1050.

13. Bohnsack, J. F., and X. Zhou. 1992. Divalent cation substitution reveals CD18- and very late antigen-dependent pathways that mediate human PMN adherence to fibronectin. J. Immunol. 149:1340-1347.

14. Bohnsack, J. F., S. K. Akiyama, C. H. Damsky, W. A. Knape, and G. A. Zimmerman. 1990. Human neutrophil adherence to laminin in vitro. Evidence for a distinct neutrophil integrin receptor for laminin. J. Exp. Med. 171:1221-1237.

15. Reinhardt, P. H., J. F. Elliot, and P. Kubes. 1997. Neutrophils can adhere via $\alpha 4 \beta 1$-Integrin under flow conditions. Blood 89:3837-3846.

16. Kubes, P., X. Niu, C. W. Smith, M. Kehrli, Jr., P. Reinhardt, and R. Woodman. 1995. A novel beta 1-dependent adhesion pathway on neutrophils: a mechanism invoked by dihydrocytochalasin B or endothelial transmigration. FASEB J. 9:1103-1111.

17. Gao, J. X., and A. C. Issekutz. 1995. Polymorphonuclear leukocyte migration through human dermal fibroblast monolayers is dependent on both beta-2 integrin (CD11/CD18) and beta-1 integrin (CD29) mechanisms. Immunology 85:485-492.

18. Gao, J. X., and Issekutz, A. C. 1997. The $\beta 1$ integrin, very late activation antigen- 4 on human neutrophils can contribute to neutrophil migration through connective tissue fibroblast barriers. Immunology 90:448-454.

19. Attibele, N., P. Wyde, J. Trial, S. Smole, C. Smith, and R. Rossen. 1993. Measles virus-induced changes in leukocyte function antigen 1 expression and leukocyte aggregation: possible role in measles virus pathogenesis. J. Virol. 67:1075-1079.

20. Bergelson, J., B. Chan, R. Finberg, and M. Hemler. 1993. The integrin VLA-2 binds echovirus 1 and extracellular matrix ligands by different mechanisms. J. Clin. Invest. 92:232-239.

21. Rao, S., K. Ogata, and A. Catanzaro. 1993. Mycobacterium aviumM. intracellulare binds to the integrin receptor alpha $\mathrm{V}$ beta 3 on human monocytes and monocyte-derived macrophages. Infect. Immun. 61:663-670.

22. Christensen, J., E. Andersson, A. Scheynius, O. Marker, and A. Thomsen. 1995. Alpha 4 integrin directs virus-activated CD8+ T cells to sites of infection. J. Immunol. 154:5293-5301.

23. Weeks, B., M. Klotman, S. Dhawan, M. Kibbey, J. Rappasport, and H. Kleinman. 1991. HIV-1 infection of human T lymphocytes results in enhanced alpha 5 beta 1 integrin expression. J. Cell Biol. 114:847-853.

24. Leblond, V., C. Legendre, G. Gras, N. Dereuddre-Bosquet, C. Lafuma, and D. Dormont. 2000. Quantitative study of beta-1 integrin expression and fibronectin interaction profile of $\mathrm{T}$ lymphocytes in vitro infected with HIV. AIDS Res. Hum. Retroviruses. 16:423-433.

25. Whalen, M. J., T. M. Carlos, R. S. Clark, D. W. Marion, M. S. Dekosky, S. Heineman, J. K. Schiding, F. Memarzadeh, C. E.
Dixon, and P. M. Kochanek. 1997. The relationship between brain temperature and neutrophil accumulation after traumatic injury in rats. Acta. Neurochir. Suppl. 70:260-261.

26. Whalen, M. J., T. M. Carlos, R. S. Clark, D. W. Marion, S. T. Dekosky, S. Heineman, J. K. Schiding, F. Memarzadeh, and P. M. Kochanek. 1997. The effect of brain temperature on acute inflammation after traumatic brain injury in rats. J. Neurotrauma 14:561-572.

27. Chatzipanteli, K., O. Alonso, S. Kraydieh, and W. Dietrich. 2000. Importance of posttraumatic hypothermia and hyperthemia on the inflammatory response after fluid percussion brain injury: biochemical and immunocytochemical studies. J. Cereb. Blood Flow Metab. 20:531-542.

28. Chatzipanteli, K., Y. Yanagawa, A. Marcillo, S. Kraydieh, R. Yezierski, and W. Dietrich. 2000. Posttraumatic hypothermia reduces polymorphonuclear leukocyte accumulation following spinal cord injury in rats. J. Neurotrauma. 17:321-332.

29. Irazuzta, J., R. Pretzlaff, M. Rowin, K. Milam, and B. Zingarelli. 2000. Hypothermia in the treatment of severe bacterial meningitis. Brain Res. (in press).

30. Irazuzta, J., J. Olson, M. Kiefaber, and H. Wong. 1999. Hypothermia decreases excitatory neurotransmitter release in bacterial meningitis in rabbits. Brain Res. 847:143-148.

31. Rowin, M., V. Xue, and J. Irazuzta. 2000. Integrin expression on neutrophils in a rabbit model of group B streptococcal meningitis. Inflammation 24:157-174.

32. Yednock, T., C. Cannon, C. Vandevert, E. Goldbach, G. Shaw, D. Ellis, C. Liaw, L. Fritz, and L. Tanner. 1995. Alpha 4 beta 1 integrin-dependent cell adhesion is regulated by a low affinity receptor pool that is conformationally responsive to ligand. J. Bio. Chem. 270:28740-28750.

33. Albelda, S. M., and C. A. Buck. 1990. Integrins and other cell adhesion molecules. FASEB J. 4:2868-2880.

34. Marcantonio, E. E., and R. Hynes. 1988. Antibodies to conserve cytoplasmic domain of the integrin beta-1 subunit react with proteins in vertebrates, invertebrates, and fungi. J. Cell Biol. 106: $1765-1772$.

35. Brower, D. L., S. M. Brower, D. C. Hayward, and E. E. Ball. 1997. Molecular evolution of integrins: genes encoding integrin beta subunits from a coral and a sponge. Proc. Natl. Acad. Sci. U.S.A. 94:9182-9187.

36. Yenari, M. A., D. Kunis, G. H. Sun, D. Onley, L. Watson, S. Turner, S. Whitaker, and G. K. Steinberg. 1998. Hu23F2G, an antibody recognizing the leukocyte $\mathrm{CD} 11 / \mathrm{CD} 18$ integrin, reduces injury in a rabbit model of transient focal cerebral ischemia. Exp. Neurol. 153:223-233.

37. Bavbek, M., R. Polin, A. L. Kwan, A. S. Arthur, N. F. Kassell, and K. S. Lee. 1998. Monoclonal antibody against ICAM-1 and CD18 attenuate cerebral vasospasm after experimental subarachnoid hemorrhage in rabbits. Stroke 29:1930-1936.

38. Rogers, C., E. R. Edelman, and D. I. Simon. 1998. A MAb to the beta 2-leukocyte integrin Mac-1 reduces intimal thickening after angioplasty or stent implantation in rabbits. Proc. Natl. Acad. Sci. U.S.A. 95:10134-10139.

39. Enomoto-Iwamoto, M., M. Iwamoto, K. Nakashima, Y. Mukudai, D. Boettiger, M. Pacifici, K. Kurisu, and F. Suzuki. 1997. Involvement of alpha5 beta1 integrin in matrix interactions and proliferation of chondrocytes. J. Bone Miner. Res. 12:1124-1132.

40. Espinosa, G., A. Lopez-Farre, M. R. Cernadas, F. Manzarbeitia, D. Tan, E. Digiuni, J. R. Mosquera, M. Monton, L. Hernando, S. Casado, and C. Caramelo. 1996. Role of endothelin in the pathophysiology of renal ischemia-reperfusion in normal rabbits. Kidney Int. 50:776-782.

41. Rowin, M., R. Pretzlaff, V. Xue, and J. Irazuzta. 1999. Role of beta 1 integrins on CSF neutrophils in a rabbit model of meningitis. Crit. Care Med. 27:129. 
42. Rowin, M., V. Xue, and J. Irazuzta. 1999. Hypothermia attenuates $\beta 1$ integrin expression on neutrophils in an animal model of meningitis. Ped. Res. 45:173.

43. Clifton, G., J. Jiang, B. Lyeth, L. Jenkins, R. Hamm, and R. Hayes. 1991. Marked protection by moderate hypothermia after experimental traumatic brain injury. J. Cereb. Blood Flow Metab. 11:114-121.

44. Dietrich, W., O. Alonso, R. Busto, M. Globus, and M. Ginsberg. 1994. Post-traumatic brain hypothermia reduces histopathologic damage following concussive brain injury in the rat. Acta Neuropathol. 87:250-258.

45. Clifton, G., S. Allen, P. Barrodale, P. Plenger, J. Berry, S. Koch, J. Fletcher, R. Hayes, and S. Choi. 1993. A phase II study of moderate hypothermia in severe brain injury. J. Neurotrauma. 10:263-271.

46. Cornejo, C., P. Kierney, N. Vedder, and R. Winn. 1998. Mild hypothermia during reperfusion reduces injury following ischemia of the rabbit ear. Shock 9:116-120.

47. Bering, E. 1961. Effect of body temperature change on cerebral oxygen consumption of the intact monkey. Am. J. Physiol. 31:417-419.

48. Marion, D., L. Penrod, S. Kelsey, W. Obrist, P. Kochanek, A. Palmer, S. Wisniewski, and S. Dekosky. 1997. Treatment of traumatic brain injury with moderate hypothermia. N.E.J.M. 336:540-546.

49. Garbulinski, T., B. O. Domoradzka, M. Switala, and J. Debowy. 1991. Responses of neutrophils and lymphocytes in cold stress: effects of nonsteroid anti-inflammatory drugs. Pol. J. Pharmacol. Pharm. 43:353-359.

50. Williams, H., N. Rebuck, M. Elliot, and A. Finn. 1998. Changes in leukocyte count and soluble intercellular adhesion molecule-1 and E-selectin during cardiopulmonary bypass in children. Perfusion 13:322-327.

51. Boldt, J., C. Osmer, L. Linke, G. Gorlach, and G. Hemplemann. 1996. Hypothermic versus normothermic cardiopulmonary bypass: influence on circulating adhesion molecules. J. Cardiothorac. Vasc. Anesth. 10:342-347.

52. Menasche, P., J. Peynet, N. Haeffner-Cavaillon, M. Carreno, T. De
Chaumaray, V. Dillisse, B. Faris, A. Piwnica, G. Bloch, and A. Tedgui. 1995. Influence of temperature on neutrophil trafficking during clinical cardiopulmonary bypass. Circulation 92:334-340.

53. Finn, A., N. Moat, N. Rebuck, N. Klein, S. Strobel, and M. Elliot. 1993. Changes in neutrophil CD11b/CD18 and L-selectin expression and release of interleukin 8 and elastase in paediatric cardiopulmonary bypass. Agents Actions 38:44-46.

54. Paugam, C., S. Chollet-Martin, M. Dehoux, D. Chatel, N. Brient, J. Desmonts, and I. Philip. 1997. Neutrophil expression of CD11b/CD18 and IL-8 secretion during northmothermic cardiopulmonary bypass. J. Cardiothorac. Vasc. Anesth. 11:575-579.

55. Hawkins, H. K., S. C. Heffelfinger, and D. C. Anderson. 1992. Leukocyte adhesion deficiency: clinical and postmortem observations. Ped. Path. 12:119-130.

56. Jaeschke, H., A. Farhood, M. A. Fischer, and C. W. Smith. 1996. Sequestration of neutrophils in the hepatic vasculature during endotoxemia is independent of $\beta 2$ integrins and intercellular adhesion molecule-1. Shock 6:351-356.

57. Doerschuk, C. M., R. K. Winn, H. O. Coxson, and J. M. Harlan. 1990. CD18-dependent and independent mechanisms of neutrophil emigration in the pulmonary and systemic microcirculation of rabbits. J. Immunol. 144:2327-2333.

58. Hellewell, P. G., S. K. Young, P. M. Henson, and G. S. Worthen. 1994. Disparate role of the $\beta 2$-integrin CD18 in the local accumulation of neutrophils in pulmonary and cutaneous inflammation in the rabbit. Am. J. Respir. Cell Mol. Biol. 10:391-398.

59. Tan, T., C. Smith, E. Hawkins, and S. Kaplan. 1997. Anti-CD11b monoclonal antibody in an infant rat model of Haemophilus influenzae type b sepsis and meningitis. J. Antimicrob. Chemother. 39:209-216.

60. Bogdan, I., S. L. Leib, M. Bergeron, L. Chow, and M. G. Tauber. 1997. Tumor necrosis factor-alpha contributes to apoptosis in hippocampal neurons during experimental group B streptococcal meningitis. J. Infect. Dis. 176:693-697.

61. Lahrtz, F., L. Piali, K. Spanaus, J. Seebach, and A. Fontana. 1998. Chemokines and chemotaxis of leukocytes in infectious meningitis. J. Neuroimmunology 85:33-43. 